\title{
Hybrid pore-network and Lattice-Boltzmann permeability modelling accelerated by machine learning
}

DOI:

10.1016/j.advwatres.2019.02.012

\section{Document Version}

Accepted author manuscript

Link to publication record in Manchester Research Explorer

\section{Citation for published version (APA):}

Rabbani, A., \& Babaei, M. (2019). Hybrid pore-network and Lattice-Boltzmann permeability modelling accelerated by machine learning. Advances in Water Resources. https://doi.org/10.1016/j.advwatres.2019.02.012

\section{Published in:}

Advances in Water Resources

\section{Citing this paper}

Please note that where the full-text provided on Manchester Research Explorer is the Author Accepted Manuscript or Proof version this may differ from the final Published version. If citing, it is advised that you check and use the publisher's definitive version.

\section{General rights}

Copyright and moral rights for the publications made accessible in the Research Explorer are retained by the authors and/or other copyright owners and it is a condition of accessing publications that users recognise and abide by the legal requirements associated with these rights.

\section{Takedown policy}

If you believe that this document breaches copyright please refer to the University of Manchester's Takedown Procedures [http://man.ac.uk/04Y6Bo] or contact uml.scholarlycommunications@manchester.ac.uk providing relevant details, so we can investigate your claim.

\section{OPEN ACCESS}




\title{
Hybrid pore-network and Lattice-Boltzmann permeability modelling accelerated by machine learning
}

\author{
Arash Rabbani ${ }^{\mathrm{a}}$, Masoud Babaei ${ }^{\mathrm{a}, *}$ \\ ${ }^{a}$ The University of Manchester, School of Chemical Engineering and Analytical Science, \\ Manchester, UK
}

\begin{abstract}
In this paper, a permeability calculation workflow is presented that couples pore network modeling (PNM) with a Lattice Boltzmann Method (LBM) to benefit from the strengths of both approaches. Pore network extraction is implemented using a watershed segmentation algorithm on 12 three-dimensional porous rock images. The permeabilities of all throats are calculated using the LBM and substituted in the pore network model instead of using the cylindrical formulation for throat's permeability based on the Hagen-Poiseuille equation. Solving the LBM for every throat results in an accurate representation of flow but the algorithm is computationally expensive. In order to minimize the computational costs, LBM is used to model the steady-state incompressible fluid flow through 9,333 different throat images and an Artificial Neural Network $(\mathrm{ANN})$ is trained to mimic the trend of throat's permeabilities based on the cross-sectional images. To this end, we extract several morphological features of the throats cross-sectional images and search for the best describing feature. It is found that the averaged distance map of the throat images is highly correlated with the LBM-based permeability of throats to the extent that even a simple empirical correlation can reasonably describe the relationship between these two parameters. Finally, we compare the absolute permeability of samples obtained by full LBM with the presented hybrid method. Results show
\end{abstract}

\footnotetext{
* Corresponding author

Email address: masoud.babaei@manchester.ac.uk (Masoud Babaei)
}

Preprint submitted to Advances in Water Resources

February 23, 2019 
that the proposed method provides an accurate estimation of permeability with a considerable reduction in the computational CPU time.

Keywords: Lattice Boltzmann Method, Pore Network Modeling, Machine Learning, Permeability, Distance Map

\section{Introduction}

2 1.1. Permeability calculation

$3 \quad$ Microscale computed tomography has triggered a series of advances in porous 4 media studies by revealing accurate interior structures non-destructively [1, 2,

5 3, 4, 5. Knowledge of microscopic porous structure provides a better under6 standing of porous media properties, for example, hydraulic conductivity or 7 absolute permeability. In many research areas from material science to hydrol8 ogy and geoscience, hydraulic conductivity and permeability of porous materials have been subjects of research during the past decades [6, 7, , 8, 9, 10, 11, 12. Existing approaches for estimating the pore-level permeability using computed tomography images can be categorized as direct and indirect methods. "Direct" refers to the fact that in these methods realistic geometry of the porous media is taken into account and governing equations are written to be solved on the exact structure. Finite Volume 13, 14, 15, 4, Finite Element 16, 17, 18, 19] and Lattice Boltzmann [20, 21, 22, 23, 24, 25, 26] are the most prominent direct simulation methods in the literature. Conversely, indirect methods do not consider the exact geometry of porous media and the equations are solved on a simplified proxy model that behaves similarly to the original geometry. Pore Network Modeling [27, 28] and Bundle-of-Tubes [29, 30, 31] are two indirect methods that have been widely used for modeling and simulation of several transport phenomena in porous media.

\subsection{Direct simulation methods}

As mentioned, these methods are able to be implemented for the exact geometry of porous media with a minimal amount of simplification. As a result, 
they could be accurate if the governing equations and boundary conditions are selected and solved properly. The main limitation of direct methods is the high computational cost [1], which could be a major obstacle when we are dealing with large-sized and high-resolution volumetric images of porous material. As a solution to this size and time limitation, domain decomposition and parallel computation have been comprehensively hired to increase the models efficiency and scalability [32, 33, 34, 35, 36. Additionally, as another solution to deal with computational limitations, machine learning can be employed to mimic the behaviour of the complex solid/fluid systems. The main idea is to save the computational sources by solving a series of typical/representative problems and extend the results to all similar cases [37, 38, 39. For this purpose, we need to break down a large problem into several small independent pieces and try to find computationally inexpensive features which are statistically related to the original problem.

As one of the most trending approaches in pore-scale flow modeling, Lattice Boltzmann method has been initiated by Frisch et al. 40 under the name of lattice-gas automata in 1986. Later in 1988, Boltzmann equation was plugged into the method proposed by McNamara and Zanetti [41] and named as LBM. This direct simulation method can be easily implemented by computer programming [42] and it's a versatile tool to consider multiple physics in a parallelized fashion [43. So, in addition to the single phase flow modelling, more advanced processes such as multiphase flow including the effect of variable wettablity can be implemented by LBM [4, 45, 46]. Classic LBM [40] uses a uniform lattice grid for simulation and this could reduce the simulation performance by avoiding local coarsening/refinement of the grid, while modern approaches have coupled the finite volume and finite element schemes with LBM calculations to increase the performance of the method by adopting multi-domain/unstructured grid which can catch more complex geometries [47, 48, 49]. However, LBM could be computationally expensive. In a comparison published by Manwart et al. [50] it was claimed that LBM demands 2.5 times more memory than the amount required by finite difference on the same porous geometry and similar CPU time. 


\subsection{Indirect methods}

Indirect methods such as pore network modeling assume several geometrical simplifications to reduce the computational costs, but lead to error in the simulation results 28, 51. As an example, in the classical pore network models, the pressure drop is neglected in pore bodies and throat cross-sections are considered as definitive geometrical shapes such as circle, square and triangle [28]. Also, in order to make the PNM's more realistic, complicated throat shapes have been investigated by coupling a series of mathematical curves and defining an analytical shape factor for throats [52. In comparison, none of these assumptions are made in the direct simulation methods and the realistic geometry with many of its morphological details are used to build a mesh and initiate the numerical simulation. Considering the relentless advances in the 3D imaging techniques [53. 54 and large size of the recorded images, low-cost computational methods such as PNM are becoming popular. However, the level of simplifications in PNM should be minimized to avoid compromising the accuracy.

\subsection{Coupling direct and indirect methods}

In recent years, there have been several efforts to couple different direct and indirect simulation methods to increase the performance of permeability models [55, 56, 57]. Miao et al. [55] extracted 3,292 pore elements and simulated the single-phase permeability of the pore elements using COMSOL Multiphysics software by solving the Navier-Stokes equation. They used 3 image-based features to train an Artificial Neural Network (ANN) as a means to predict the throat absolute permeability as well as shape factor, solidity, and aspect ratio of the images. In a similar but improved approach, here, we couple both direct and indirect methods for calculation of porous media permeabilities. Furthermore, in addition to features extracted by Miao et al. [55], we study four more imagebased features from 9,333 pore-throats and substitute the throat permeabilities in a pore network model to calculate the overall permeability of rock samples. 


\subsection{Outline}

The rest of the paper is organized as follows. Section 2 describes the proposed new methodology to couple LBM and PNM methods to model porous material permeability. In this section we also describe the studied porous rock samples. In Section 3 we present the results of pore network extraction, statistical analyses of the extracted throat features, results of machine learning application, empirical correlations derived for throat's permeability along a physical justification. Finally, calculated permeabilities and computational costs compared to conventional methods are presented. The paper is concluded in Section 4.

\section{Methodology}

\subsection{Synopsis}

Based on the definition, pore throat is referred to the locations of the porous elements in which the opening of the pathway reaches to its minimum. Thus, the permeability of such tight openings play as a bottleneck for the rest of the structure and will be critical in determination of the macroscopic properties.

As opposed to the classical pore network modeling that describes throat cross-sections analytically [5, 52, we assemble a workflow to handle any arbitrary throat shape with minimal computational costs. As an illustration, a 3D image of Berea Sandstone is modeled and absolute hydraulic permeability is calculated with both classical and proposed PNM-LBM methods (Fig. 17). As we will show in Section 3 the PNM-LBM (Fig. 1 $1 \mathrm{~b}$ ) method can calculate the absolute permeability of the porous samples with a higher accuracy than the classical PNM (Fig. 11). 


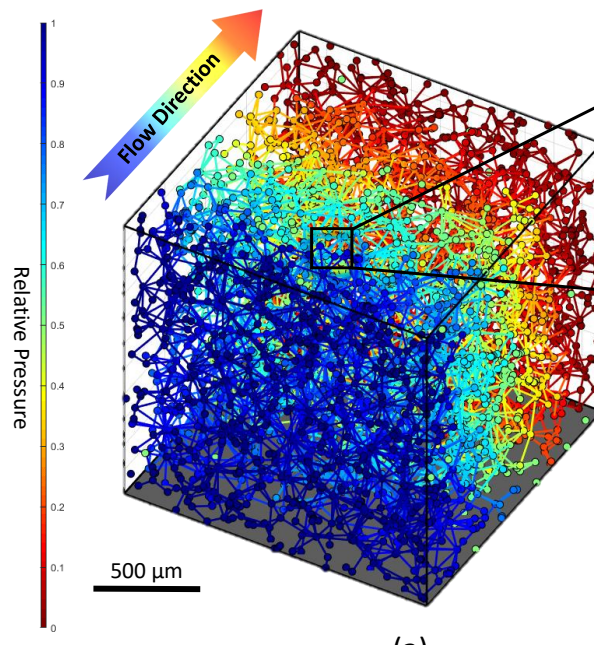

(a)

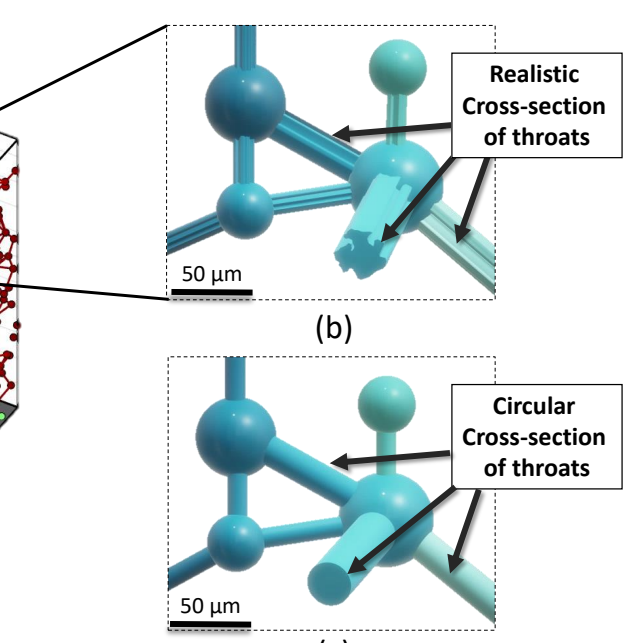

(c)

Figure 1: Visual comparison of modified (PNM-LBM) and classical PNM approaches, (a) single-phase steady-state pressure drop in a pore network of Berea Sandstone with relative pressures between 0 and 1, (b) the PNM-LBM approach with realistic throat cross-sections, and (c) the classical PNM approach with circular throat cross-sections.

In this study, instead of the hydraulic radius, we use D3Q19 Lattice Boltzmann modeling with Bhatnagar-Gross-Krook (BGK) approach [58] to estimate the throat's permeability. In order to reduce the computational costs of LBM simulations, we introduce Image-Based Throat Permeability Model (ITPM) $)^{1}$ The workflow of building this model is described step by step in Fig. 2, The process starts with preparation of micro-CT images including noise filtering and binarization. Next, the pore network is extracted by help of the watershed algorithm and cross-sectional image of throats are recorded in a database. Additionally, several morphological features of the throat images are extracted and stored in the database. Next, we run a series of relatively time consuming LBM simulations on throat images to determine their absolute permeability and

\footnotetext{
${ }^{1}$ Image-Based Throat Permeability Model is available in the Supplementary information as well as on on GitHub: https://github.com/ArashRabbani/ITPM
} 
relate them to the permeabilities of the database. Using the database and by training two ANN's and one empirical correlation we build a set of ITPM's that are able to predict the permeability of the throats with minimal computational cost.

Next, ITPM is used to find the hydraulic properties of the pore networks in a hybrid approach that couples both PNM and LBM. In this regards, Fig. 3 presents the simplified workflow in a stepwise manner. A 3-D binary pore-level image is taken as an input of the workflow. At the first step, we need to extract the pore network. This is done in a subsidiary workflow (Fig. 3a) comprising noise removal, distance transform, Gaussian filtering, watershed segmentation, labeling and connectivity detection. A brief description of pore network extraction is presented in the next subsection. The extracted pore network is used in the next step of the main workflow (Fig. 3b) for feature extraction from throat cross-sections. This step, similarly is shown in a subsidiary workflow (Fig. 3c) and comprises of projecting 3-D image of throat surfaces on a 2-D plane and then calculating the average value of its distance map. The extracted feature goes back to the main workflow as an input for ITPM. This process should be repeated for all throats of the network. Finally, the ITPM permeabilities are substituted in the classical PNM. 


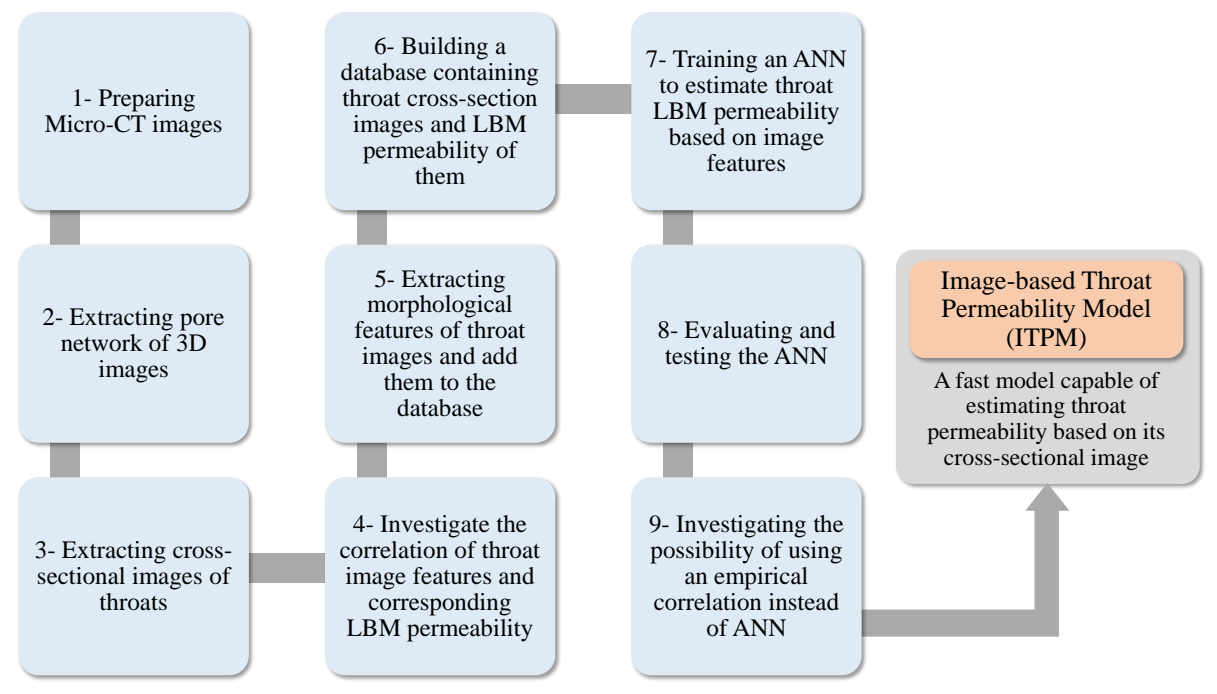

Figure 2: Workflow of building the Image-Based Throat Permeability Model (ITPM).

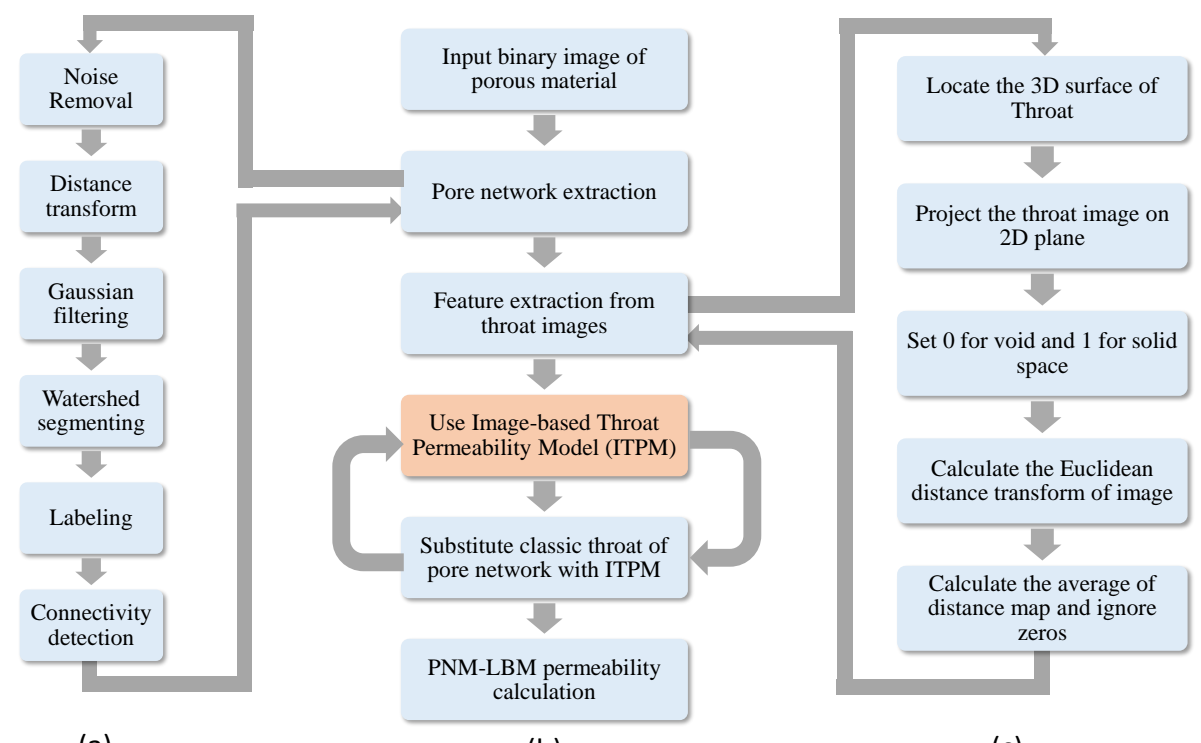

(a)

(b)

(c)

Figure 3: Workflow of the hybrid PNM-LBM approach for calculation of the pore-level permeability, (a) subsidiary workflow of pore network extraction, (b) main workflow of the PNMLBM permeability calculation, and (c) subsidiary workflow of throat features extraction. 


\subsection{Pore network extraction}

A brief procedure for extracting pore networks is illustrated in Fig. 3(a). Watershed segmentation algorithm is used for pore network extraction. This method is computationally efficient and sufficiently accurate for auto-detection of pore bodies and pore throats [59, 60, 61, 62]. Watershed algorithm utilizes the distance transform of the pore space to find out the narrowest pathways of porous media and introduces them as network throats [63, 64, 65]. In order to have more stabilized results and avoid over-segmentation, 3D Gaussian filtering is implemented on the distance transform [59, 66. The output of the watershed segmentation is an image with isolated segments each of which represents a unique pore body. This image goes under labeling process to identify all isolated spaces. Finally, by searching the whole image voxel by voxel, we detect and record adjacent pore spaces with unique labels to build a connectivity matrix which represents the extracted pore network. In this workflow of pore network extraction, throats are curved surfaces at the interface of two adjacent pores (Fig. 4 4). In this study, we do not simplify the throat cross-section to circles and preserve their original shape to compute a more realistic permeability using a Lattice Boltzmann method. We extract the throat surfaces along the line that connects the centers of two adjacent pores and forms a prism with arbitrary base shape (Fig. 4b). 


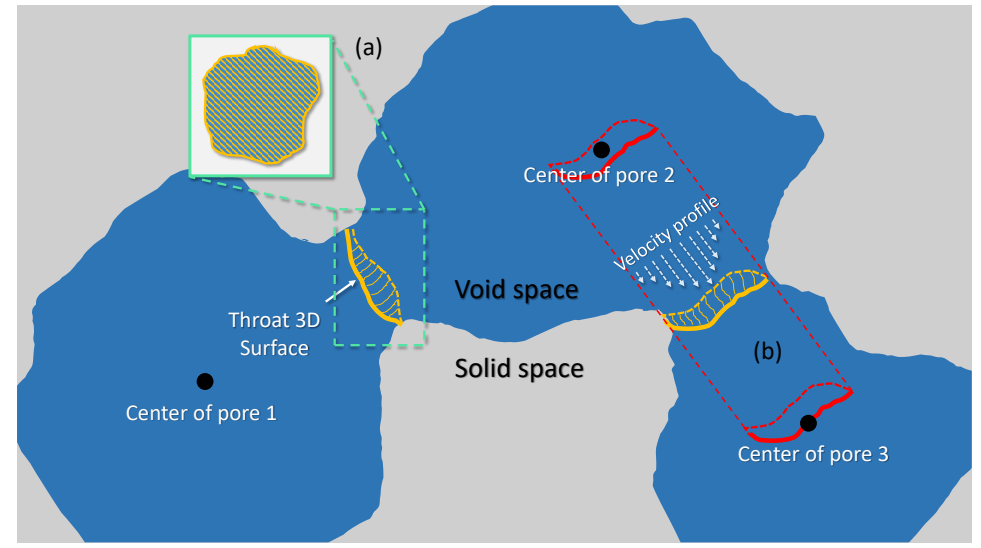

Figure 4: Throats definition in the model, (a) a projected image of 3-D throat surface on 2-D plane which is used for feature extraction, and (b) extruded geometry of the throat cross-section which is considered as the flow pathway between the pore centers.

\subsection{Extraction of features}

As mentioned, throats are considered to be the interfaces where two adjacent pores touch each other 63. These interfaces may have slight curvatures based on the morphology of the original volumetric image of porous media. In order to effectively extract the image features, we project the throat images in the direction of a line that connects the centers of two adjacent pores. Thus, 3D slightly curved surfaces will be converted to flattened 2D binary images which represent the cross-section of the tightest pathway between two adjacent pores (Fig. 4a). Next, we use MATLAB Image Processing Toolbox to extract multiple morphological features that may be correlated with hydraulic conductivity of the throats.

Table 1 presents a list of features we extract from 2-D images of throats in addition to their definitions.

Although most of these features have been extensively discussed in the literature for characterization of porous structures. Amongst these, average distance map needs more clarifications. Thus, consider a throat with circular crosssection as shown in Fig. 5(a). Assign zero to the void space and one to the solid space of the image. Then instead of each zero pixel, put the Euclidean distance 
between that pixel and the nearest non-zero pixel in the image (Fig. 5 b). Consequently, this distance value will be equal to zero for all the pixels located in the solid space. This transform is known as the distance transform and it is available in most of major open-source or commercial image processing packages. The final feature that we are using to correlate with the throat's LBM-based permeability is "non-zero average of the distance map". A physical justification will be presented in Section 3 to explain the relationship between this feature and the throat permeabilities.

\begin{tabular}{|c|c|c|}
\hline No. & Feature & Feature Description \\
\hline 1 & Cross-section area $\left(\right.$ pixel $\left.^{2}\right)$ & Surface area of the $2-\mathrm{D}$ projection of throat \\
\hline 2 & Wetted perimeter (pixel) & Perimeter of the $2-\mathrm{D}$ projection of throat \\
\hline 3 & Axes ratio & $\begin{array}{l}\text { Ratio between the major and minor axes of the } \\
\text { throat which is always greater than } 1\end{array}$ \\
\hline 4 & Equivalent diameter (pixel) & $\begin{array}{l}\text { Diameter of the circle with the same area as } \\
\text { the throat }\end{array}$ \\
\hline 5 & Solidity & $\begin{array}{l}\text { Area of the throat convex hull divided by the } \\
\text { throat area }\end{array}$ \\
\hline 6 & Hydraulic radius (pixel) & $\begin{array}{l}\text { Throat area multiplied by two divided by } \\
\text { throat wetted perimeter }\end{array}$ \\
\hline 7 & Mean distance (pixel) & $\begin{array}{l}\text { Non-zero average of the throat distance trans- } \\
\text { form }\end{array}$ \\
\hline
\end{tabular}

Table 1: Extracted throat features and their description.

\begin{tabular}{|c|c|c|c|c|c|c|c|c|c|c|}
\hline & & & & & & & \multirow{2}{*}{\multicolumn{3}{|c|}{ 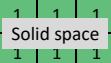 }} & \\
\hline & 1 & 1 & 1 & 0 & 0 & 0 & & & & \\
\hline & 1 & & & & 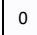 & 0 & & 0 & 1 & \\
\hline & 1 & 0 & 10 & & 0 & 0 & & 0 & & \\
\hline & 0 & 0 & 0 & 0 & c & & & 0 & & \\
\hline & 0 & & & & (1) & & & 0 & & \\
\hline & & & & & 0 & 0 & 0 & 0 & & \\
\hline & & & & 10 & 0 & 0 & 0 & 0 & 1 & \\
\hline & 1 & 0 & 0 & & & & 0 & & & \\
\hline & 1 & 1 & 1 & 0 & 0 & 0 & 1 & 1 & 1 & 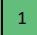 \\
\hline & 1 & & & & & & & 1 & & \\
\hline
\end{tabular}

(a)

\begin{tabular}{|c|c|c|c|c|c|c|c|c|c|c|}
\hline 0 & 0 & 0 & 0 & 0 & 0 & 0 & 0 & 0 & 0 & 0 \\
\hline 0 & 0 & 0 & 0 & 1 & 1 & 1 & 0 & 0 & 0 & 0 \\
\hline 0 & 0 & 1 & 1 & 1.4 & 2 & 1.4 & 1 & 1 & 0 & 0 \\
\hline 0 & 0 & 1 & 2 & 2.2 & 2.8 & 2.2 & 2 & 1 & 0 & 0 \\
\hline 0 & 1 & 1.4 & 2.2 & 3.2 & 3.6 & 3.2 & 2.2 & 1.4 & 1 & 0 \\
\hline 0 & 1 & 2 & 2.8 & $3.6-4.5$ & 3.6 & 2.8 & 2 & 1 & 0 \\
\hline 0 & 1 & $1.4-2-2.2$ & 3.2 & 3.6 & 3.2 & 2.2 & 1.4 & 1 & 0 \\
\hline$-2-0$ & -0 & 1 & 2 & 2.2 & 2.8 & 2.2 & 2 & 1 & 0 & 0 \\
\hline 0 & 0 & 1 & 1 & 1.4 & 2 & 1.4 & 1 & 1 & 0 & 0 \\
\hline 0 & 0 & 0 & 0 & 1 & 1 & 1 & 0 & 0 & 0 & 0 \\
\hline 0 & 0 & 0 & 0 & 0 & 0 & 0 & 0 & 0 & 0 & 0 \\
\hline
\end{tabular}

(b)

Figure 5: Definition of the distance transform on a circular cross-section, (a) throat crosssection in which zero indicates the void space and one indicates the solid space, (b) values are equal to the Euclidean distance between the current pixel and the nearest non-zero pixel. 


\subsection{Throat flow simulation with the LBM}

In order to model single-phase steady-state flow in a throat, we consider it as a tube with an arbitrary cross-section with a uniform shape along the throat length. We assume that the single-phase flow in throats is steady-state and fully developed. So, technically it is not required to simulate the whole length of the throat from the center of one pore to the center of the adjacent pore to find out its permeability (Fig. 6a). We take the $2 \mathrm{D}$ cross-section of a throat (Fig. 6p) and stack 6 layers of that cross-section (Fig. 6d) to build the simulation geometry. A D3Q19 scheme (Fig. 6c) is considered for the LBM which means there are 18 possible directions for fluid flow within each block. Considering the small size of the $2 \mathrm{D}$ throat images, image voxels are considered to be the simulation blocks without any upscaling.

We adopt an open-source LBM code in MATLAB to predict the permeability of the tube section (throat), originally developed by Haslam et al. [67]. A Newtonian fluid with BGK collision model [58] is used. Also, it is assumed that bounce-back is only in the direction normal to the geometry boundary. Particle distribution function $f_{i}$ evolves in the directions of the distribution vectors $e_{i}$ at each time step $(t+\Delta t)$ and location $(x)$ as [67]:

$$
f_{i}\left(x+e_{i}+\Delta t\right)-f_{i}(x, t)=-1 / \tau\left(f_{i}(x, t)-f_{i}^{e q}(x, t)\right)
$$

where $f_{i}^{e q}$ is a truncated Maxwell-Boltzmann equilibrium distribution that can be expressed as a function of local velocity $(v)$ in all possible directions and $\tau$ is equilibrium state time. Also $i$ is the distribution function index for different neighbours. Equilibrium function is [67]:

$$
f_{i}^{e q}=u w_{i}\left(1+3 e_{i} \cdot v\right)
$$

where $w_{i}$ indicates neighbour weights and its values are $1 / 3,1 / 18$, and $1 / 36$ for stationary, nearest and next-nearest vectors, respectively (Fig. 6r).

We start the flow simulation by imposing uniform distribution vectors (velocities) to the inlet blocks of the geometry. Periodic boundary condition is 
assumed in the direction of flow which means that during the LBM simulation, velocity distribution in the outlet face of geometry are assigned to the geometry inlet velocity distribution. It is known that the absolute permeability of tubes in laminar flow is calculated as $k=r^{2} / 8$ 68, in which $r$ is the tube radius. Considering that this formulation is independent of the tube length, periodic boundary condition is a valid assumption for the geometry considered.

The LBM simulation continues until a convergence is reached in the magnitude and direction of the distribution vectors at each block. This equilibrium state can be expressed in terms of permeability convergence by keeping the relative error smaller than a specified value. The convergence criterion we use to detect the density equilibrium is

$$
\text { error }=\frac{k_{\text {old }}-k_{n e w}}{k_{\text {old }}}<10^{-6}
$$

where $k_{\text {old }}$ and $k_{n e w}$ are two consecutive values obtained for permeability during iterations. In order to calculate the permeability of throat tubes at each iteration, Darcy's law is rearranged as:

$$
k=-\frac{\mu \bar{U}}{\frac{d p}{d x}}
$$

where $\bar{U}$ is the averaged velocity vector in the direction of pressure drop, $k$ is absolute permeability, $d p / d x$ is the pressure gradient that similar to the velocity vector is obtained from LBM simulation results, and $\mu$ is the fluid viscosity which is calculated as:

$$
\mu=\frac{\frac{1}{\omega}-0.5}{3}
$$

where $\omega$ is a relaxation frequency used in LBM. We assume that it is equal to 1 to ensure convergence of the LBM densities and minimize the error [69, 70].

After running several time steps in an explicit manner, permeability values converge to a value which is recorded as the absolute permeability of the simulated throat geometry. As an illustration, the permeability calculation method 
discussed above is applied on some throats' images with arbitrary shapes. Velocity maps at the outlet surface are visualized in Fig. 7. In this figure, sections (a) to (f) present the exiting velocity maps for throats with circular, starshape, square, triangular, sample convex and sample concave cross-sections, respectively. In all images, as it was expected, velocity has higher values where node is farther away from the throat wall. Also, for the circular cross-section (Fig. 7a), the analytical and numerical permeabilities match with the value of $25 \mu \mathrm{m}^{2}$.

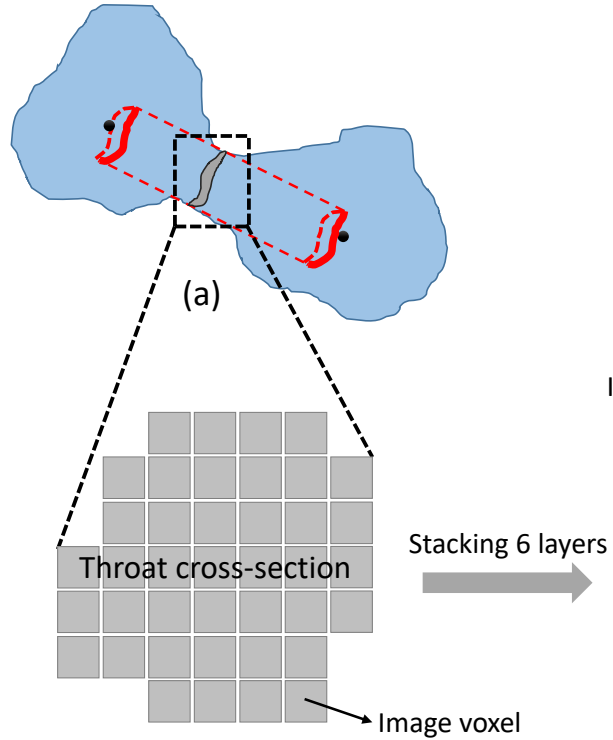

(b)

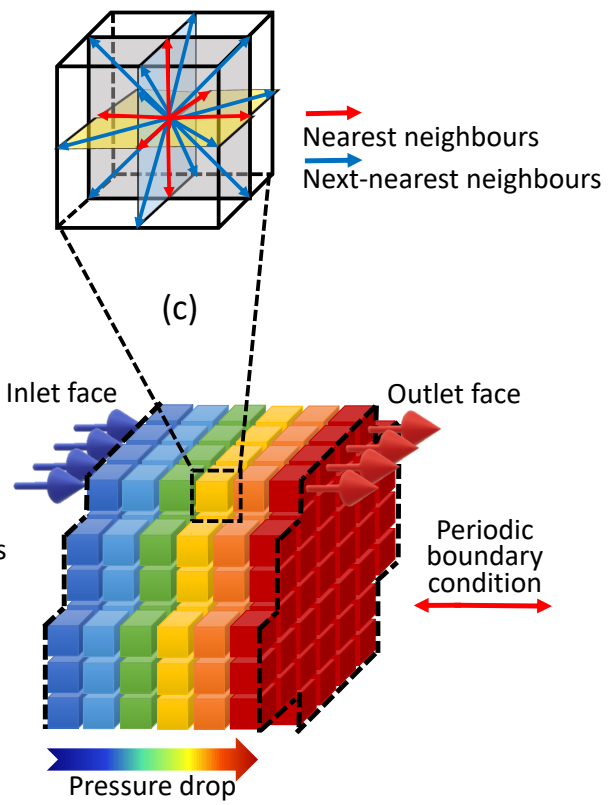

(d)

Figure 6: Extraction and construction of the equivalent throat geometry used for LBM simulation, (a) equivalent throat geometry used in the pore network model and its cross-section, (b) cross-section of the throat acquired from image processing, (c) D3Q19 scheme of LBM comprised of nearest and next-nearest neighbour vectors, and (d) schematic pressure drop during the flow and periodic boundary condition in the direction of flow. 


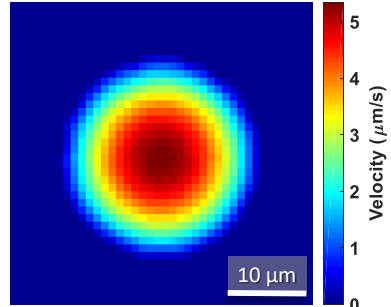

(a)

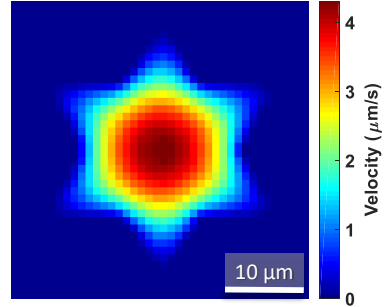

(b)

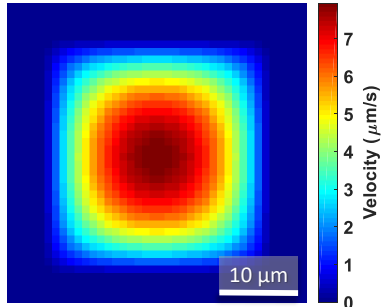

(c)

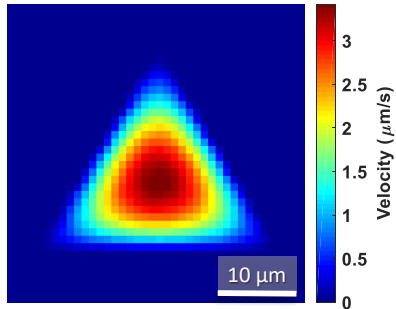

(d)

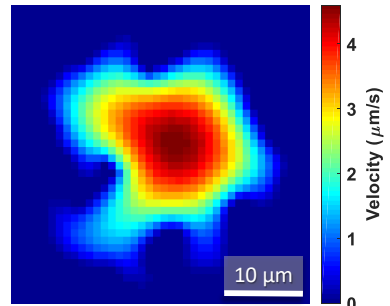

(e)

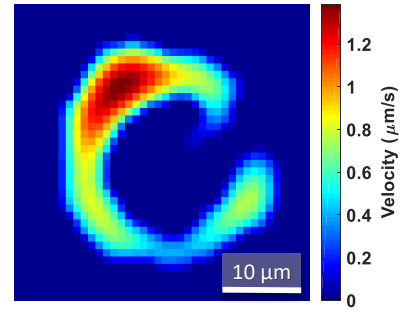

(f)

Figure 7: Exiting velocity map for different throat cross-sections obtained by LBM, (a) circular, (b) star-shape, (c) square, (d) triangular, (e) arbitrary and convex, and (f) arbitrary and concave cross-section.

\subsection{Machine learning}

We use machine learning to avoid repetitive calculation of the LBM-based permeability in all throats. Machine learning methods inherently classify the input samples into self-similar categories and develop a unique matrix-based relationship for each category to predict the best accurate outputs [71]. In this study, we use Levenberg-Marquardt method to train two ANN's in order to predict the throat's permeability based on the image-extracted features. Levenberg-Marquardt is an iterative algorithm to find the minimum value of a function that depends on the sum of squares of a series of nonlinear functions [72]. This algorithm is a common tool to optimize weights and biases of an ANN in a reasonable time scale [73. From the two trained ANNs, the first ANN (Fig. 8a) takes 7 input parameters and has 6 nodes in the hidden layer and one output parameter. The input parameters are cross-sectional area, wetted perimeter, axes ratio, equivalent diameter, solidity, hydraulic radius, and mean distance. In order to avoid over-fitting, size of the hidden layer is set -based 
on a rule of thumb- equal to the $2 / 3$ of input parameters plus the number of output parameters. Neural network training is performed using the functions existing in Neural Fitting Toolbox of MATLAB.

The second ANN (Fig. 8p) is simpler and composed of one input parameter which is the mean distance, three nodes in the hidden layer and one output parameter. We train the second ANN to check out the effect of omitting probably less significant input parameters and present a simpler model.

In order to train these ANN's, we build a database composed of 9,333 entries using the throat images extracted from 12 porous samples used in this study (Table 2).

From total number of analyzed throats, we take $65 \%$ of them for training, $15 \%$ for validation and $20 \%$ for testing. Then mean squared error (MSE) and coefficient of determination $\left(R^{2}\right)$ are calculated to check the performance of training, validation and testing processes. Using the trained networks, we are able to calculate the throat's permeability without running the LBM. 
(a)

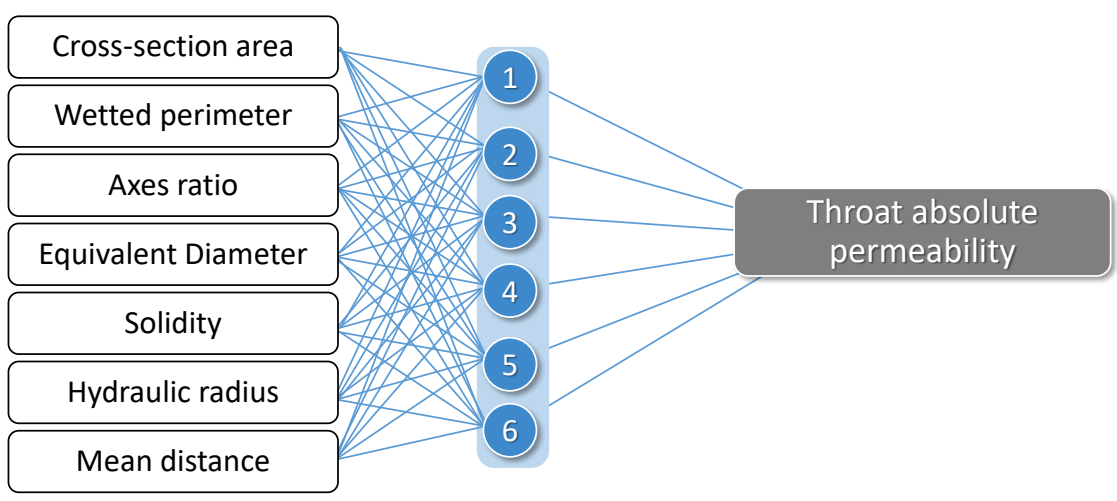

(b)

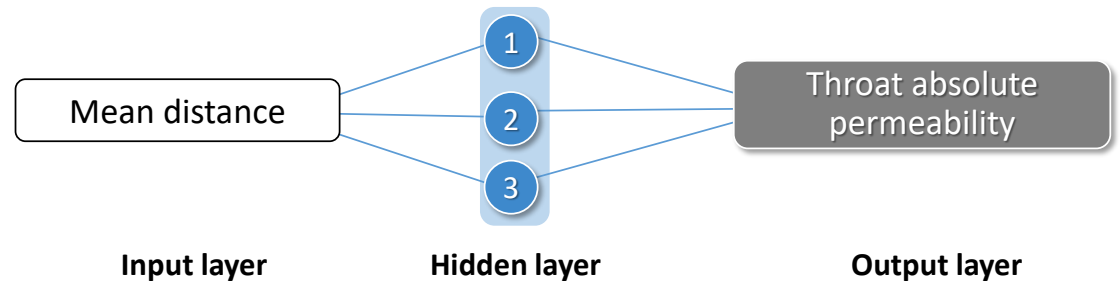

Figure 8: Illustration of two ANN's employed to model the permeability of throats based on the image features and previously calculated LBM-based permeabilities which are stored in a database, (a) the first ANN with 7 features as input and hidden layer with 6 nodes, and (b) the second ANN with 1 feature as input and 3 nodes in the hidden layer.

\subsection{Empirical correlation for throat's permeability}

Machine learning is typically applied in systems with complicated/non-linear relationships between the inputs and outputs [74. ANN's are powerful and versatile tools for these purposes, however, they cannot be easily transferred to or replicated by other researchers, as they would need all nodal weights and biases. Thus, we aim to present an empirical correlation which relates the throat's LBM-based permeability and the best fitted feature which is extracted from throat images. A general form will be considered for the correlation based on the trial and error. Then using an optimization method we minimize the error of prediction by assigning values to the correlation constants. The optimized correlation will be presented in Section 3 . 


\subsection{Samples studied}

In this study, we use 12 micro-CT images to investigate the permeability prediction accuracy of the proposed method. The samples are imaged at Imperial College London [5, 75, and their size and resolution are listed in Table 2 Samples $\mathrm{C} 1$ and $\mathrm{C} 2$ are carbonate rocks and rest of the samples are sandstone including Br sample (Berea Sandstone). These samples have been thoroughly studied in literature [76, 1, 63, 75, 77.

\begin{tabular}{l|l|l}
\hline Name & Resolution $(\mu \mathrm{m} /$ voxel $)$ & Size (voxel) \\
\hline S1 & 8.68 & $300^{3}$ \\
S2 & 4.96 & $300^{3}$ \\
S3 & 9.10 & $300^{3}$ \\
S4 & 8.96 & $300^{3}$ \\
S5 & 4.00 & $300^{3}$ \\
S6 & 5.10 & $300^{3}$ \\
S7 & 4.80 & $300^{3}$ \\
S8 & 4.89 & $300^{3}$ \\
S9 & 3.40 & $300^{3}$ \\
C1 & 2.85 & $400^{3}$ \\
C2 & 5.35 & $400^{3}$ \\
Br & 5.35 & $400^{3}$ \\
\hline \multicolumn{2}{|l|}{ Table 2: List of studied porous rock samples. }
\end{tabular}

In order to avoid the effect of different spatial image resolutions, we temporarily change all spatial image resolutions to one micrometer per pixel. Then, after calculation of throat's permeability, we undo this change by multiplying the obtained value by the second power of the real spatial resolution provided in Table 2 ,

\section{Results and Discussions}

\subsection{Pore network extraction}

Pore network extraction is implemented on 12 porous samples (Table 2) studied in this research to provide the throat images needed for the LBM-based permeability simulation. In addition, these networks will be used to calculate 
the total permeability of the samples by assuming throat permeabilities derived by ITPM. The properties of extracted pore networks are provided in Table 3 .

The throat radius reported is obtained by assuming equivalent circles with same surface area. In the current modeling, the pore radius of the network does not directly affect network permeability. Pore radius is just used to calculate and match the network porosity. Also, we have not taken into the account the pressure drop of pores. This is because of the fact that pressure drop in porous elements are mainly controlled by throats which are the narrowest openings rather than the pore bodies. In the extracted networks, we define the length of throats as the distance between centroids of two adjacent pores.

\begin{tabular}{l|l|l|l|l}
\hline Samples & $\begin{array}{l}\text { Mean pore ra- } \\
\text { dius }(\mu \mathrm{m})\end{array}$ & $\begin{array}{l}\text { Mean throat } \\
\text { radius }(\mu \mathrm{m})\end{array}$ & $\begin{array}{l}\text { Mean throat } \\
\text { length }(\mu \mathrm{m})\end{array}$ & $\begin{array}{l}\text { Mean pore con- } \\
\text { nectivity }\end{array}$ \\
\hline S1 & 49.18 & 24.88 & 207.04 & 2.64 \\
S2 & 30.64 & 16.22 & 120.13 & 3.80 \\
S3 & 32.55 & 14.26 & 143.84 & 2.49 \\
S4 & 32.68 & 15.49 & 133.83 & 2.07 \\
S5 & 26.07 & 21.85 & 128.81 & 2.45 \\
S6 & 33.33 & 26.73 & 145.57 & 3.23 \\
S7 & 35.11 & 18.95 & 138.38 & 4.11 \\
S8 & 27.16 & 21.24 & 126.60 & 3.56 \\
S9 & 29.91 & 17.57 & 115.83 & 3.00 \\
C1 & 11.39 & 11.99 & 62.49 & 2.12 \\
C2 & 18.00 & 15.10 & 94.29 & 1.55 \\
Br & 26.22 & 14.70 & 112.98 & 2.90 \\
\hline
\end{tabular}

Table 3: Properties of the classical pore networks extracted using watershed segmentation algorithm.

\subsection{Features statistics}

Total number of 9,333 throats are analyzed from 12 extracted networks and 7 morphological features are extracted from each throat image. This database includes a wide range of porous rocks with diverse fabric and morphological properties. We have statistically analyzed these features to measure the dependency of the permeability on each of them. In this line, stepwise regression coefficients of the features are calculated and shown in Table 4 relative to the 
absolute permeability. Stepwise regression is an automated method for fitting regression models and find the explanatory variables [78]. A higher regression coefficient means that the function value is more correlated to the corresponding variable [78. According to Table 4 , it can be concluded that mean distance is the best correlated variable. Also, the hydraulic radius of the throat is reasonably correlated to the LBM-based permeability. Considering the higher correlation of mean distance, we have selected this feature to be used as single input of the second ANN. In addition, the proposed empirical correlation for throat's permeability will utilize the mean distance as input variable to have the best performance in permeability estimation.

Fig. 9 illustrates the scatter plots of 7 image-extracted features vs. absolute permeabilities. In order to remove the effect of different image resolutions, we consider "pixel" unit for length and "pixel" for area. Thus, in these figures permeability values are shown with "pixel" ${ }^{2}$ " unit. By visual comparison of the scatter plots from Fig. 9 (a) to (g), it can be concluded that absolute permeability of throats has a more significant relationship with the mean distance (Fig $9 \mathrm{~s}$ ). This conclusion is previously verified considering the higher value of stepwise regression coefficient corresponding to mean distance (Table 4 ).

\begin{tabular}{l|l}
\hline Feature & Stepwise regression coefficient \\
\hline Cross-section area & 0.017 \\
Wetted perimeter & 0.013 \\
Axes ratio & -0.003 \\
Equivalent Diameter & -0.627 \\
Solidity & -1.391 \\
Hydraulic radius & 0.572 \\
Mean distance & 4.399
\end{tabular}

Table 4: Stepwise regression coefficients between the extracted features of throats and their corresponding LBM-based permeability. 


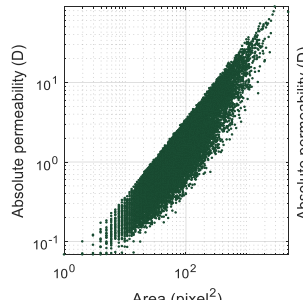

(a)

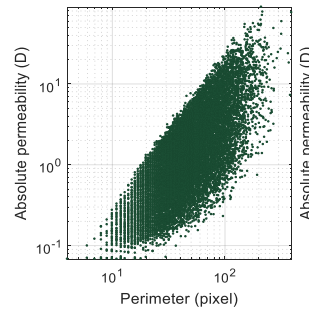

(b)

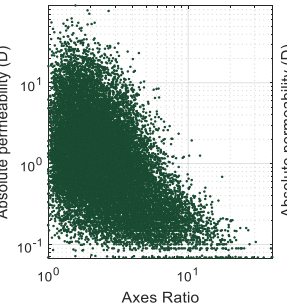

(c)

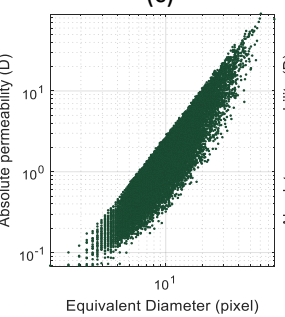

(d)

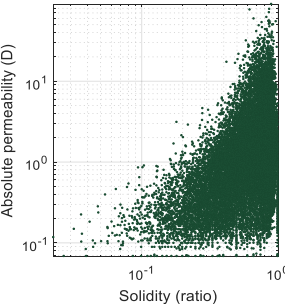

(e)

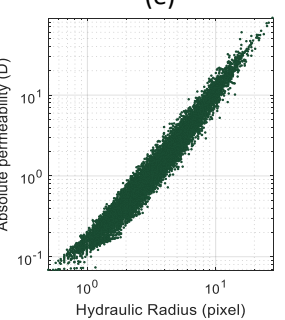

(f)

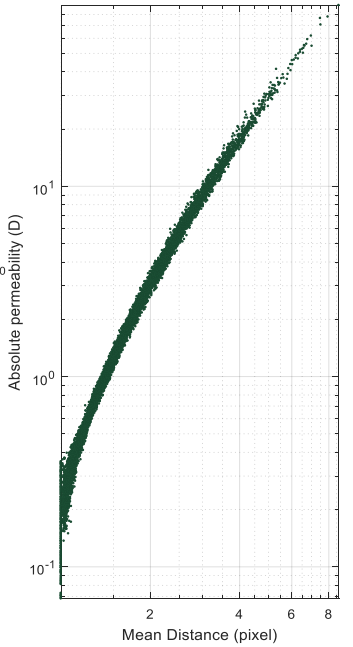

(g)

Figure 9: Scatter plot of LBM absolute permeability vs. values of 7 extracted features of throats: (a) cross-sectional area, (b) wetted perimeter, (c) axes ratio, (d) equivalent radius, (e) solidity, (f) hydraulic radius, and (g) mean distance.

\subsection{Machine learning}

Visual comparison between the cross-plots of Fig. 9 leads to the mean distance as the selected well-correlated input parameter to be used for the singleparameter ANN. The points of Fig. 9p are less scattered and apparently follow a predictable trend. Both 7- and single-parameter ANN's are trained to be able to predict throat's permeability. The learning performance of both ANN's are provided in Table 5 in terms of mean squared error (MSE) and coefficient of determination $\left(R^{2}\right)$. Although, generally higher number of input parameters will enhance the ANN's predictions, parsimony and simplicity of the model will be affected. Consequently, the single-parameter ANN is able to estimate the LBMbased permeability with a reasonably high coefficient of determination (Testing $R^{2}=0.9982$ ), as such it would be more practical to use single-parameter ANN than to use the 7-parameter ANN.

Fig. 10(a) and (b) illustrate the original and predicted throat permeabilities by single- and 7-parameter ANN's, respectively. As it is expected, the 


\begin{tabular}{l|l|l|l|l}
\hline Data Set & 7-Param. MSE & 7-Param. $R^{2}$ & 1-Param. MSE & 1-Param. $R^{2}$ \\
\hline Training & 0.0142 & 0.9998 & 0.1436 & 0.9984 \\
Validating & 0.0193 & 0.9997 & 0.1281 & 0.9980 \\
Testing & 0.0264 & 0.9996 & 0.1971 & 0.9982 \\
\hline
\end{tabular}

Table 5: Learning performance of both 7- and single-parameter ANN's in terms of coefficient of determination $\left(R^{2}\right)$ and mean squared error (MSE).

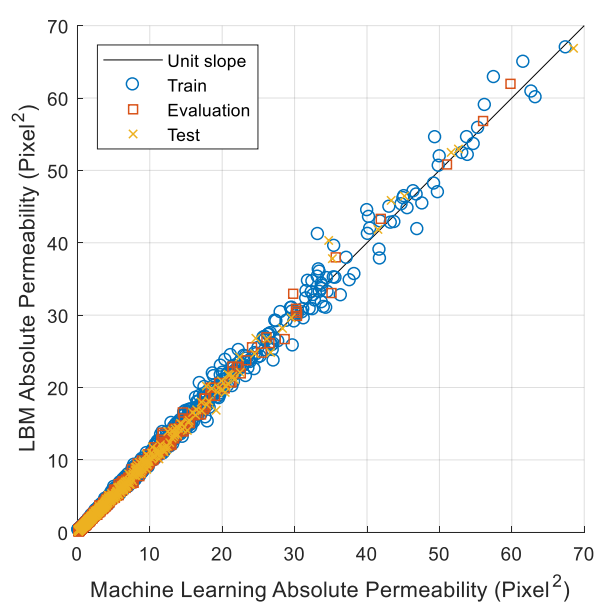

(a)

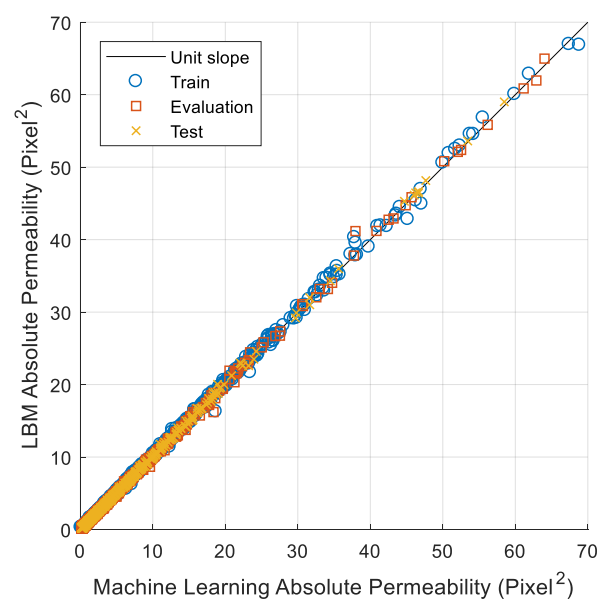

(b)

Figure 10: The capability of ANN's to predict LBM-based permeability of throats including training, evaluation and testing data, (a) single-parameter ANN, (b) 7-parameter ANN.

predictions of single-parameter ANN is more scattered than the 7-parameter ANN. 
where $k_{\text {throat }}$ is throat's permeability with pixel $^{2}$ unit, and $\bar{D}$ is mean distance of the throat image. Optimization of the correlation constants is carried out by linear least squares method. The constants obtained for the empirical formula and the $95 \%$ confidence interval for those constants are provided in Table 6. Fig 11 illustrates the original and estimated LBM-based permeabilities using the proposed formula. The overall $R^{2}$ and MSE of the empirical correlation results are 0.996 and 0.146 , respectively. These values are in the same order of magnitude as $R^{2}$ and MSE of single-parameter ANN. Thus it is reasonable to use the proposed empirical correlation when ANN's are not available. Finally, the proposed empirical formula for throat's permeability can be written as:

$$
k_{\text {throat }}=1.342 \bar{D}^{2}-0.913 \bar{D}-0.381
$$

\begin{tabular}{l|l|l|l}
\hline Model constants & Mean value & $\begin{array}{l}\text { Lower bound- } \\
\text { ary }\end{array}$ & $\begin{array}{l}\text { Upper bound- } \\
\text { ary }\end{array}$ \\
\hline$a$ & 1.342 & 1.338 & 1.345 \\
$b$ & -0.9127 & -0.9376 & -0.8878 \\
$c$ & -0.3814 & -0.4131 & -0.3498 \\
\hline
\end{tabular}

Table 6: Constants in the empirical formula and their $95 \%$ confidence intervals.

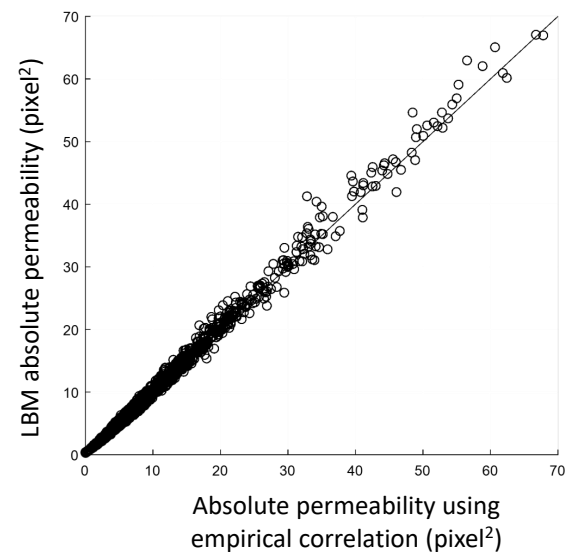

Figure 11: Original LBM-based throat permeabilities and predicted values using the proposed empirical formula $\left(R^{2}=0.996\right)$. 


\subsection{Physical justification}

We showed statistically that mean distance is the best image feature to estimate the LBM-based permeability of a throat. Here, we explain the physical concept behind this observation. Based on the distance map definition, we know that the distance value of a pixel in a void space is higher for the pixel that is far from the solid walls (Fig. 5p. Similarly, for a steady-state incompressible laminar flow through a tube, fluid velocity reaches its maximum at the center of the tube since fluid is far from the tube walls (Fig. 77). So, distance map mimics the trend of the velocity profiles in the tubular laminar flow. In order to illustrate this statement, Fig. 12 is presented. The top row of the figure, shows the relative velocity maps obtained from the LBM simulations and the bottom row is the relative distance map of the throat cross-sections. For the sake of comparison, we have divided all values of both map types over their maximum values to generate the maps with relative intensities. In addition, the non-zero average and non-zero standard deviation of both groups of maps are inscribed in the figure. Based on these three pairs of examples, the difference is not more than $25 \%$ between the LBM velocity maps and the distance maps. By the term "none-zero average" we mean that the zero values (dark blue portion of the maps) are not considered in averaging. 


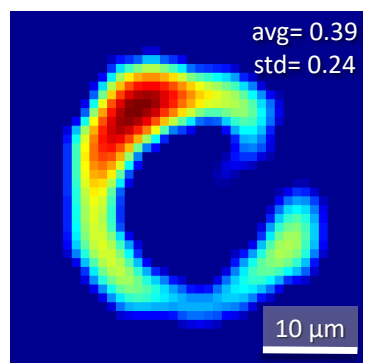

(a)

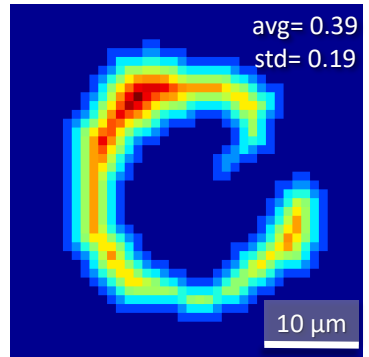

(b)

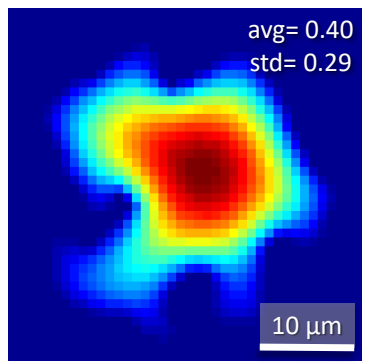

(c)

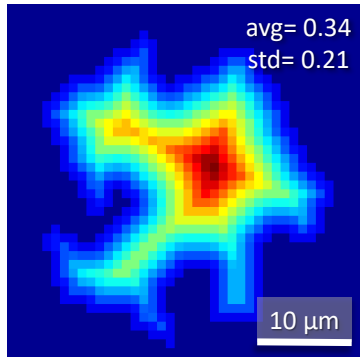

(d)

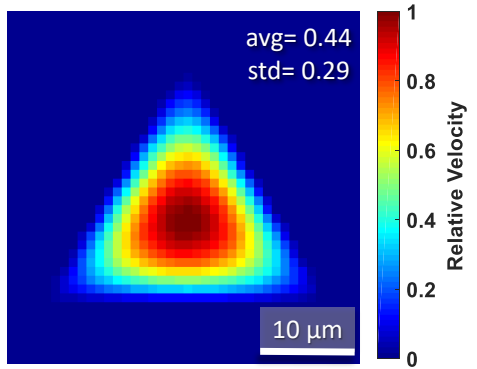

(e)

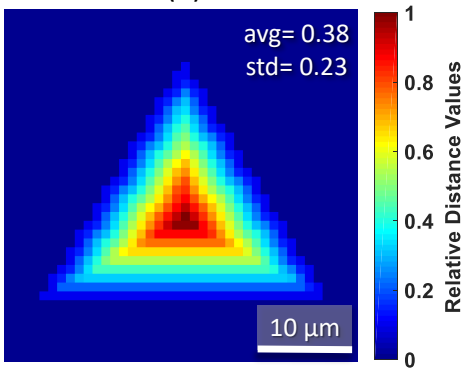

(f)

Figure 12: Comparison of velocity maps and distance maps obtained by the LBM simulation in three different throats: (a) and (b) concave cross-sections, (c) and (d) convex cross-sections, (e) and (f) triangular cross-sections.

\subsection{Permeability calculation}

When the permeability of all network throats is estimated using ITPM, there is one step remaining to calculate the permeability of the whole pore network.

For this step, we assume a pressure difference between two sides of the network so that the pressure at the center of the pores are unknown. In the next step, the continuity equation is written for each pore for a steady-state single-phase flow of an incompressible fluid. Then we rearrange the continuity equation in term of throat's permeability, length and pore pressures. Since throat's permeability and length are previously known, the only unknown parameters are the pressure of each pore. This leads to a linear system of equation which can be numerically solved to find the pressures. After determination of all pressures, the overall flow rate is calculated and total absolute permeability of the network is obtained using Darcy's law. We call the final value "hybrid PNM-LBM permeability" 
since both methods are used. Here, we compare three different options for throat's permeability calculation and compare the total permeability of our 12 samples for each option. The three options for throat's permeability are as follow:

\section{a) Hagen-Poiseuille permeability with equivalent radius}

In this option, throat's permeability is calculated as $r^{2} / 8$ in which $r$ is throat radius and it is equal to the radius of a circle with the same surface area as the throat.

\section{b) Hagen-Poiseuille permeability with hydraulic radius}

In this option, throat's permeability is calculated as $r^{2} / 8$ in which $r$ is hydraulic radius of the throat and can be calculated as $2 A / P$, that $A$ is throat cross area and $P$ is the wetted perimeter of throat.

\section{c) LBM-based permeability}

In this option, permeability of throats is calculated using the LBM simulation and by means of Eq. 7 .

In addition, the total permeability of the studied samples when running LBM on the whole geometry is available in the literature [76. Consequently, we are able to compare our calculated permeabilities with total LBM-based permeability of the samples. This comparison is illustrated in Fig. 13 by averaging the absolute permeability of samples in $x, y$ and $z$ directions. Fig. 13(a) (for Option a) shows the PNM permeability considering equivalent radius for throats vs. the total LBM-based permeability of the sample. It is clear that the predicted values are over-estimated. This could be due to ignoring the wetted perimeter of the throats in permeability calculation. Larger wetted perimeter causes more friction during fluid flow and reduces the throat's permeability. As an example, angular throat shapes are less permeable than the circular ones, although they have the same cross-sectional area. Fig. 13(b) presents results for Option b. The difference is that for this option, we are calculating the throat's permeability using the hydraulic radius concept and this is a more realistic approach than op- 


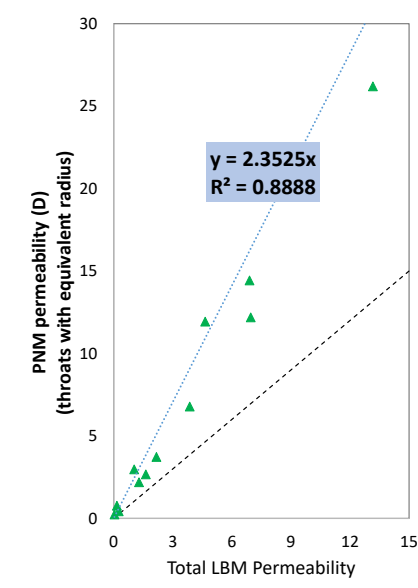

(a)

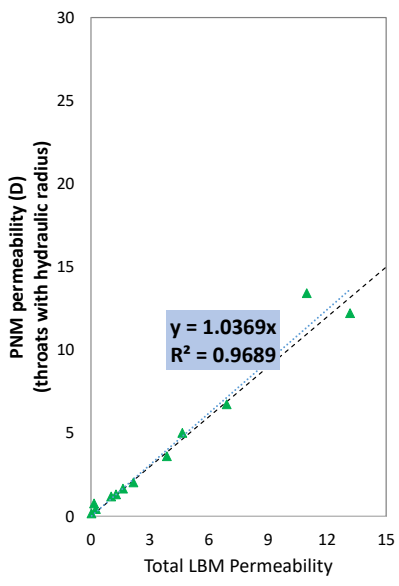

(b)

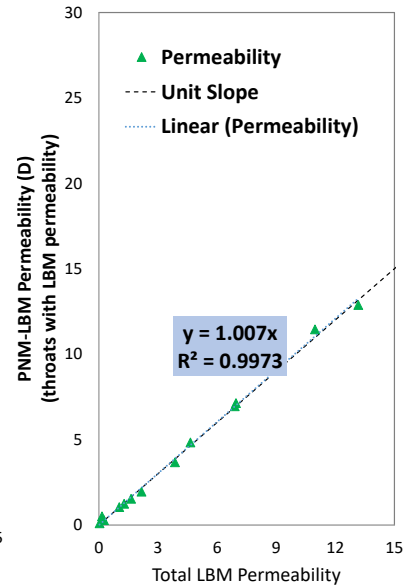

(c)

Figure 13: Comparison of network permeability values if using three different options for throat's permeability, (a) throats with permeability based on the equivalent radius concept, (b) throats with permeability based on the hydraulic radius concept, and (c) throats with permeability estimated using LBM by means of Eq. 7

\subsection{Computational costs}

Here, we present the CPU times of the simulation machine while running different permeability modelling techniques. The results are presented in Fig. 14 CPU times of the full LBM are adopted from Mostaghimi et al.[76] who performed LBM on a sandpack image (LV60) with spatial resolution of 10 micrometer per voxel. CPU times of the classical PNM and the PNM-LBM methods are measured in the present study. All simulations are performed using a $3.0 \mathrm{GHz}$ CPU with no parallel processing. As it can be seen in Fig. 14 the PNM-LBM 
method is not computationally efficient and its CPU time is increasing with a steep trend relative to the other methods. The accelerated PNM-LBM which uses Eq. 7 for the throat's permeability, is virtually as fast as the classical PNM approach but as we have shown in Fig. 13, it is more accurate. It is noteworthy that for the PNM-based methods, CPU time of pore network extraction is included in total times. Finally, it can be stated that using the CPU configuration mentioned and with PNM-LBM approach, the absolute permeability of a sample (in the size of $400^{3}$ voxels) can be calculated in around 200 seconds.

In addition to the CPU time saving, the PNM-LBM approach demands less computational memory. The amount of peak memory usage for full LBM-based permeability model on the discussed $300^{3}$ sample is $3.5 \mathrm{~GB}$. For the tested PNM-based methods, memory needed is around 1 GB when running on the same sample. Additionally, it is possible for the PNM-based methods to use even less amount of memory if using domain decomposition approach for running pore network extraction. This part of the presented code has been shown to be a memory bottleneck 35 .

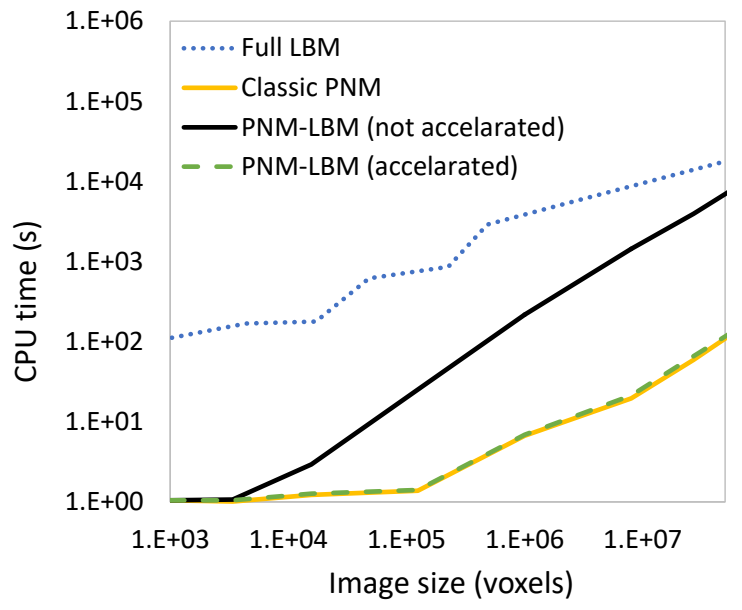

Figure 14: CPU times of the different methods for calculating the absolute permeability of a sandpack image [76. Tested methods are full LBM (CPU times from [76]), the classical PNM 61], the PNM-LBM without machine learning acceleration, and the PNM-LBM with machine learning acceleration. 


\section{Conclusions}

In this study, we coupled PNM and LBM approaches to estimate the hydraulic permeability of porous rock samples. In this regards, a classic pore network model is extracted from micro-tomography images and throat permeabilities are simulated using LBM. Then, in order to reduce the computational cost of the LBM simulation, we utilized some image-based features to estimate LBM throat permeabilities in an efficient manner.

The main conclusions and findings of this study are as follow:

- Two ANN's and one empirical formula were presented capable of estimating the throat's LBM-based permeabilities with coefficient of determination $\left(R^{2}\right)$ higher than 0.99 .

- The "mean distance" was found to be an image-based feature highly correlated with LBM throat permeabilities. It was defined as non-zero average of the throat distance map.

- The empirical quadratic formula for estimating the throat's LBM-based permeability employed mean distance as the input variable and this simple equation predicted the throat's permeability with coefficient of determination around 0.996 .

- The throat permeabilities obtained were substituted within the classical pore networks of 12 rock samples and overall network permeabilities were calculated successfully. We compared the PNM-LBM absolute permeabilities with pure LBM-based permeabilities of the samples and found that the proposed model was capable of predicting the overall permeability of the rock samples with coefficient of determination around 0.997.

- The proposed PNM-LBM approach was capable of predicting the permeability with higher accuracy than the classical PNM and with considerably less computational cost than the classic LBM. 


\section{Acknowledgement}

The authors thank the University of Manchester for the President's Doctoral Scholarship Award 2018 awarded to Arash Rabbani to carry out this research. We also appreciate the constructive comments of anonymous reviewers of this manuscript.

\section{References}

\section{References}

[1] M. J. Blunt, B. Bijeljic, H. Dong, O. Gharbi, S. Iglauer, P. Mostaghimi, A. Paluszny, C. Pentland, Pore-scale imaging and modelling, Advances in Water Resources 51 (2013) 197-216. doi:10.1016/j.advwatres.2012. 03.003

[ [2] D. A. Nield, A. Bejan, Convection in porous media, 2013. doi:10.1007/ 978-1-4614-5541-7.

[3] R. Hilfer, Review on scale dependent characterization of the microstructure of porous media, Transport in Porous Media 46 (2-3) (2002) 373-390. doi: 10.1023/A : 1015014302642 .

[4] A. Q. Raeini, M. J. Blunt, B. Bijeljic, Direct simulations of two-phase flow on micro-CT images of porous media and upscaling of pore-scale forces, Ad-

1. vances in Water Resources 74 (2014) 116-126. doi:10.1016/j .advwatres. 2014.08 .012

[5] H. Dong, Micro-CT imaging and pore network extraction, Ph.D. thesis, Department of Earth Science and Engineering, Imperial College London (2008).

[6] S. Jaganathan, H. Vahedi Tafreshi, B. Pourdeyhimi, A realistic approach for modeling permeability of fibrous media: 3-D imaging coupled with CFD simulation, Chemical Engineering Science 63 (1) (2008) 244-252. doi: $10.1016 / j$.ces.2007.09.020 
[7] S. Sharma, D. A. Siginer, Permeability Measurement Methods in Porous Media of Fiber Reinforced Composites, Applied Mechanics Reviews 63 (2) (2010) 020802. doi:10.1115/1.4001047.

[8] M. J. Blunt, Flow in porous media - Pore-network models and multiphase flow (2001). doi:10.1016/S1359-0294(01) 00084-X.

[9] A. J. Katz, A. H. Thompson, Quantitative prediction of permeability in porous rock, Physical Review B 34 (11) (1986) 8179-8181. doi:10.1103/ PhysRevB.34.8179.

[10] A. Szymkiewicz, Modelling water flow in unsaturated porous media: Accounting for nonlinear permeability and material heterogeneity, Geo-

Planet: Earth and Planetary Sciences 9 (2013) 1. doi:10.1007/ 978-3-642-23559-7.

[11] G. W. Jackson, D. F. James, The permeability of fibrous porous media, The Canadian Journal of Chemical Engineering 64 (3) (1986) 364-374. doi:10.1002/cjce.5450640302.

[12] A. Koponen, M. Kataja, J. Timonen, Permeability and effective porosity of porous media, Physical Review E 56 (3) (1997) 3319-3325. doi:10.1103/ PhysRevE.56.3319.

[13] Y. S. Song, K. Chung, T. J. Kang, J. R. Youn, Prediction of permeability tensor for three dimensional circular braided preform by applying a finite volume method to a unit cell, Composites Science and Technology 64 (1011) (2004) 1629-1636. doi:10.1016/j.compscitech.2003.11.008

[14] S. Geiger, S. Roberts, S. K. Matthäi, C. Zoppou, A. Burri, Combining finite element and finite volume methods for efficient multiphase flow simulations in highly heterogeneous and structurally complex geologic media, Geofluids 4 (4) (2004) 284-299. doi:10.1111/j.1468-8123.2004.00093.x.

[15] A. Q. Raeini, B. Bijeljic, M. J. Blunt, Modelling capillary trapping using finite-volume simulation of two-phase flow directly on micro-CT im- 
ages, Advances in Water Resources 83 (2015) 102-110. doi:10.1016/j. advwatres.2015.05.008.

[16] A. Munjiza, The combined finite-discrete element method, 2004. doi: $10.1002 / 0470020180$

[17] Z. Sun, R. E. Logé, M. Bernacki, 3D finite element model of semi-solid permeability in an equiaxed granular structure, Computational Materials Science 49 (1) (2010) 158-170. doi:10.1016/j.commatsci.2010.04.042

[18] J. A. White, R. I. Borja, J. T. Fredrich, Calculating the effective permeability of sandstone with multiscale lattice Boltzmann/finite element simulations, Acta Geotechnica 1 (4) (2006) 195-209. doi:10.1007/ s11440-006-0018-4.

[19] C. Sandino, P. Kroliczek, D. D. McErlain, S. K. Boyd, Predicting the permeability of trabecular bone by micro-computed tomography and finite element modeling, Journal of Biomechanics 47 (12) (2014) 3129-3134. doi:10.1016/j.jbiomech.2014.06.024.

[20] M. A. A. Spaid, F. R. Phelan, Lattice Boltzmann methods for modeling microscale flow in fibrous porous media, Physics of Fluids 9 (9) (1997) 2468-2474. doi:10.1063/1.869392.

[21] L. Hao, P. Cheng, Lattice Boltzmann simulations of anisotropic permeabilities in carbon paper gas diffusion layers, Journal of Power Sources 186 (1) (2009) 104-114. doi:10.1016/j.jpowsour.2008.09.086

[22] L. Chen, L. Zhang, Q. Kang, H. S. Viswanathan, J. Yao, W. Tao, Nanoscale simulation of shale transport properties using the lattice Boltzmann method: permeability and diffusivity, Scientific Reports 5 (2015) 8089. doi:10.1038/srep08089.

[23] C. Manwart, U. Aaltosalmi, A. Koponen, R. Hilfer, J. Timonen, LatticeBoltzmann and finite-difference simulations for the permeability for three- 
dimensional porous media, Physical Review E - Statistical, Nonlinear, and Soft Matter Physics 66 (1). doi:10.1103/PhysRevE.66.016702.

[24] E. S. Boek, M. Venturoli, Lattice-Boltzmann studies of fluid flow in porous media with realistic rock geometries, Computers and Mathematics with Applications 59 (7) (2010) 2305-2314. doi:10.1016/j.camwa.2009.08. 063.

[25] Y. Keehm, Permeability prediction from thin sections: 3D reconstruction and Lattice-Boltzmann flow simulation, Geophysical Research Letters 31 (4) (2004) L04606. doi:10.1029/2003GL018761.

[26] M. L. Stewart, A. L. Ward, D. R. Rector, A study of pore geometry effects on anisotropy in hydraulic permeability using the lattice-Boltzmann method, Advances in Water Resources 29 (9) (2006) 1328-1340. doi: 10.1016/j.advwatres.2005.10.012

[27] M. J. Blunt, M. D. Jackson, M. Piri, P. H. Valvatne, Detailed physics, predictive capabilities and macroscopic consequences for pore-network models of multiphase flow, Advances in Water Resources 25 (8-12) (2002) 10691089 .

[28] H. Dong, S. Fjeldstad, S. Roth, S. Bakke, P. Øren, Pore Network Modelling on Carbonate: A comparative Study of Different Micro-CT Network Extraction Methods, Proc. International Symp. Society of Core Analysts (2008) 2008-2031.

[29] H. K. Dahle, M. A. Celia, S. M. Hassanizadeh, Bundle-of-tubes model for calculating dynamic effects in the capillary-pressure-saturation relationship, Transport in Porous media 58 (1-2) (2005) 5-22.

[30] E. C. Childs, N. Collis-George, The permeability of porous materials, Proc. R. Soc. Lond. A 201 (1066) (1950) 392-405.

[31] F. A. Dullien, Porous media: fluid transport and pore structure, Academic press, 2012. 
[32] Y. Wang, T. Chung, R. Armstrong, J. McClure, P. Mostaghimi, Computations of permeability of large rock images by dual grid domain decomposition, Advances in Water Resources 126 (2019) 1-14.

[33] P. Mostaghimi, R. T. Armstrong, A. Gerami, Y. Hu, Y. Jing, F. Kamali, M. Liu, Z. Liu, X. Lu, H. L. Ramandi, et al., Pore scale characterisation of coal: an unconventional challenge, in: Abu Dhabi International Petroleum Exhibition \& Conference, Society of Petroleum Engineers, 2016.

[34] M. Liu, P. Mostaghimi, Pore-scale modelling of CO2 storage in fractured coal, International Journal of Greenhouse Gas Control 66 (2017) 246-253.

[35] A. Rabbani, P. Mostaghimi, R. T. Armstrong, Pore network extraction using geometrical domain decomposition, Advances in Water Resources 123 (2019) 70 - 83. doi:https://doi.org/10.1016/j.advwatres.2018. 11.003

[36] M. Liu, P. Mostaghimi, Numerical simulation of fluid-fluid-solid reactions in porous media, International Journal of Heat and Mass Transfer 120 (2018) 194-201.

[37] D. N. Hieu, T. T. Minh, T. Van Quang, B. X. Giang, T. Van Hoai, A Machine Learning-Based Approach for Predicting the Execution Time of CFD Applications on Cloud Computing Environment, in: International Conference on Future Data and Security Engineering, 2016, pp. 40-52.

[38] L. Itu, S. Rapaka, T. Passerini, B. Georgescu, C. Schwemmer, M. Schoebinger, T. Flohr, P. Sharma, D. Comaniciu, A machine-learning approach for computation of fractional flow reserve from coronary computed tomography, Journal of Applied Physiology 121 (1) (2016) 42-52.

[39] J. H. Van Der Linden, G. A. Narsilio, A. Tordesillas, Machine learning framework for analysis of transport through complex networks in porous, granular media: A focus on permeability, Physical Review E 94 (2). doi: 10.1103/PhysRevE.94.022904. 
[40] U. Frisch, B. Hasslacher, Y. Pomeau, Lattice-gas automata for the NavierStokes equation, Physical Review Letters 56 (14) (1986) 1505.

[41] G. R. McNamara, G. Zanetti, Use of the Boltzmann equation to simulate lattice-gas automata, Physical Review Letters 61 (20) (1988) 2332.

[42] S. Chen, G. D. Doolen, Lattice Boltzmann method for fluid flows, Annual review of fluid mechanics 30 (1) (1998) 329-364.

[43] S. Freudiger, J. Hegewald, M. Krafczyk, A parallelisation concept for a multi-physics Lattice Boltzmann prototype based on hierarchical grids, Progress in Computational Fluid Dynamics, an International Journal 8 (14) (2008) $168-178$.

[44] J. Zhao, Q. Kang, J. Yao, H. Viswanathan, R. Pawar, L. Zhang, H. Sun, The effect of wettability heterogeneity on relative permeability of two-phase flow in porous media: A lattice boltzmann study, Water Resources Research 54 (2) (2018) 1295-1311.

[45] A. Fakhari, Y. Li, D. Bolster, K. T. Christensen, A phase-field Lattice Boltzmann model for simulating multiphase flows in porous media: Application and comparison to experiments of $\mathrm{CO} 2$ sequestration at pore scale, Advances in Water Resources 114 (2018) 119-134.

[46] Z. Li, S. Galindo-Torres, G. Yan, A. Scheuermann, L. Li, A Lattice Boltzmann investigation of steady-state fluid distribution, capillary pressure and relative permeability of a porous medium: Effects of fluid and geometrical properties, Advances in Water Resources 116 (2018) 153-166.

[47] D. V. Patil, K. Lakshmisha, Finite volume TVD formulation of Lattice Boltzmann simulation on unstructured mesh, Journal of Computational Physics 228 (14) (2009) 5262-5279.

[48] S. Geller, M. Krafczyk, J. Tölke, S. Turek, J. Hron, Benchmark computations based on Lattice-Boltzmann, finite element and finite volume methods for laminar flows, Computers \& Fluids 35 (8-9) (2006) 888-897. 
[49] J. Yi, H. Xing, Finite element lattice boltzmann method for fluid flow through complex fractured media with permeable matrix, Advances in Water Resources 119 (2018) 28-40.

[50] C. Manwart, U. Aaltosalmi, A. Koponen, R. Hilfer, J. Timonen, Latticeboltzmann and finite-difference simulations for the permeability for threedimensional porous media, Physical Review E 66 (1) (2002) 016702.

[51] S. Bakke, P.-E. Øren, A, 3-D Pore-Scale Modelling of Sandstones and Flow Simulations in the Pore Networks, Society of Petroleum Engineers 2 (June) (1997) 136-149. doi:SPE35479.

[52] V. Joekar-Niasar, M. Prodanović, D. Wildenschild, S. Hassanizadeh, Network model investigation of interfacial area, capillary pressure and saturation relationships in granular porous media, Water Resources Research $46(6)$.

[53] S. R. Stock, Recent advances in X-ray microtomography applied to materials, International Materials Reviews 53 (3) (2008) 129-181. doi: $10.1179 / 174328008 \times 277803$

[54] T. Bultreys, M. A. Boone, M. N. Boone, T. De Schryver, B. Masschaele, L. Van Hoorebeke, V. Cnudde, Fast laboratory-based micro-computed tomography for pore-scale research: Illustrative experiments and perspectives on the future, Advances in Water Resources 95 (2016) 341-351. doi:10.1016/j.advwatres.2015.05.012.

[55] X. Miao, K. M. Gerke, T. O. Sizonenko, A new way to parameterize hydraulic conductances of pore elements: A step towards creating pore-networks without pore shape simplifications, Advances in Water Resources doi:10.1016/j .advwatres.2017.04.021

[56] T. B. Costa, K. Kennedy, M. Peszynska, Hybrid three-scale model for evolving pore-scale geometries, Computational Geosciencesdoi:10.1007/ s10596-018-9733-9. 
[57] W.-C. Sun, T.-f. Wong, Prediction of permeability and formation factor of sandstone with hybrid Lattice Boltzmann/finite element simulation on microtomographic images, International Journal of Rock Mechanics and Mining Sciences 106 (2018) 269-277.

[58] E. G. Flekkoy, Lattice Bhatnagar-Gross-Krook models for miscible fluids, Physical Review E 47 (6) (1993) 4247-4257. doi:10.1103/PhysRevE.47. 4247.

[59] J. T. Gostick, Versatile and efficient pore network extraction method using marker-based watershed segmentation, Physical Review E 96 (2) (2017) 23307.

[60] A. Rabbani, S. Ayatollahi, Comparing three image processing algorithms to estimate the grain-size distribution of porous rocks from binary $2 \mathrm{~d}$ images and sensitivity analysis of the grain overlapping degree, Special Topics \& Reviews in Porous Media: An International Journal 6 (1) (2015) 71-89. doi:10.1615/SpecialTopicsRevPorousMedia.v6.i1.60

[61] A. Rabbani, S. Ayatollahi, R. Kharrat, N. Dashti, Estimation of 3-D pore network coordination number of rocks from watershed segmentation of a single 2-D image, Advances in Water Resources 94 (2016) 264-277. doi: $10.1016 / j$.advwatres.2016.05.020

[62] D. Wildenschild, A. P. Sheppard, X-ray imaging and analysis techniques for quantifying pore-scale structure and processes in subsurface porous medium systems, Advances in Water Resources 51 (2013) 217-246.

[63] A. Rabbani, S. Jamshidi, S. Salehi, An automated simple algorithm for realistic pore network extraction from micro-tomography images, Journal of Petroleum Science and Engineering 123 (2014) 164-171.

[64] A. P. Sheppard, R. M. Sok, H. Averdunk, Improved pore network extraction methods, in: International Symposium of the Society of Core Analysts, Vol. 2125, 2005, pp. 1-11. 
[65] J.-Y. Arns, A. P. Sheppard, C. H. Arns, M. A. Knackstedt, A. Yelkhovsky, W. V. Pinczewski, Pore-level validation of representative pore networks obtained from micro-ct images, in: Proceedings of the International Symposium of the Society of Core Analysts, 2007, pp. 1-12.

[66] A. Rabbani, T. Baychev, S. Ayatollahi, A. Jivkov, Evolution of Pore-Scale Morphology of Oil Shale During Pyrolysis: A Quantitative Analysis, Transport in Porous Media 119 (1). doi:10.1007/s11242-017-0877-1.

[67] I. W. Haslam, R. S. Crouch, M. Seaïd, Coupled finite element-Lattice Boltzmann analysis, Computer Methods in Applied Mechanics and Engineering 197 (51-52) (2008) 4505-4511.

[68] N. A. Mortensen, F. Okkels, H. Bruus, Reexamination of Hagen-Poiseuille flow: Shape dependence of the hydraulic resistance in microchannels, Physical Review E - Statistical, Nonlinear, and Soft Matter Physics 71 (5). doi:10.1103/PhysRevE.71.057301

[69] U. Aaltosalmi, Fluid flow in porous media with the Lattice-Boltzmann method, no. 3, 2005.

[70] A. Pazdniakou, P. M. Adler, Dynamic permeability of porous media by the Lattice Boltzmann method, Advances in Water Resources 62 (2013) 292-302. doi:10.1016/j.advwatres.2013.06.001.

[71] E. Alpaydın, Introduction to machine learning, Vol. 1107, 2014. doi:10. 1007/978-1-62703-748-8-7.

[72] M. I. Lourakis, et al., A brief description of the levenberg-marquardt algorithm implemented by levmar, Foundation of Research and Technology 4 (1) (2005) 1-6.

[73] H. Yu, B. M. Wilamowski, Levenberg-marquardt training, Industrial Electronics Handbook 5 (12) (2011) 1. 
[74] N. M. Nasrabadi, Pattern recognition and machine learning, Journal of Electronic Imaging 16 (4) (2007) 049901.

[75] H. Dong, M. J. Blunt, Pore-network extraction from micro-computerizedtomography images, Physical Review E - Statistical, Nonlinear, and Soft Matter Physics 80 (3). doi:10.1103/PhysRevE.80.036307.

[76] P. Mostaghimi, M. J. Blunt, B. Bijeljic, Computations of absolute permeability on micro-ct images, Mathematical Geosciences 45 (1) (2013) 103125.

[77] B. Bijeljic, A. Raeini, P. Mostaghimi, M. J. Blunt, Predictions of nonfickian solute transport in different classes of porous media using direct simulation on pore-scale images, Physical Review E 87 (1) (2013) 013011.

[78] R. B. Bendel, A. A. Afifi, Comparison of stopping rules in forward "stepwise" regression, Journal of the American Statistical Association 72 (357) (1977) 46-53. 